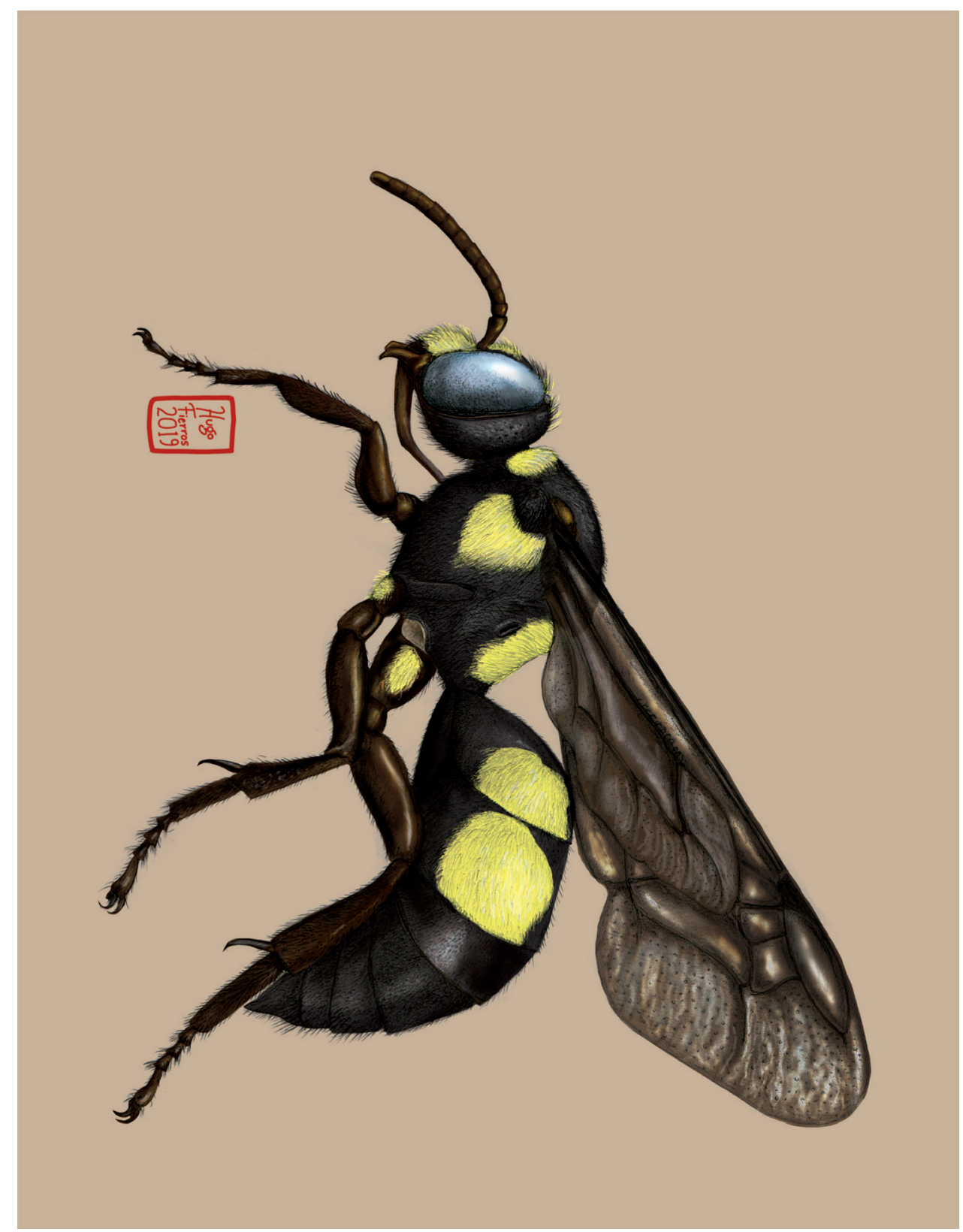

Dugesiana, Año 27, No. 1, enero 2020-junio 2020 (primer semestre de 2020), es una publicación semestral, editada por la Universidad de Guadalajara, a través del Centro de Estudios en Zoología, por el Centro Universitario de Ciencias Biológicas y Agropecuarias. Camino Ramón Padilla Sánchez \# 2100, Nextipac, Zapopan, Jalisco, Tel. 37771150 ext. 33218, http://148.202.248.171/dugesiana/index.php/DUG/index, glenusmx@gmail.com. Editor responsable: José Luis Navarrete-Heredia. Reserva de Derechos al Uso Exclusivo 04-2009-062310115100-203, ISSN: 2007-9133, otorgados por el Instituto Nacional del Derecho de Autor. Responsable de la última actualización de este número: José Luis Navarrete-Heredia, Editor y Ana Laura González-Hernández, Asistente Editorial. Fecha de la última modificación 1 de enero 2020, con un tiraje de un ejemplar.

Las opiniones expresadas por los autores no necesariamente reflejan la postura del editor de la publicación.

Queda estrictamente prohibida la reproducción total o parcial de los contenidos e imágenes de la publicación sin previa autorización de la Universidad de Guadalajara. 
Nota científica

\title{
Primeros registros de los géneros Archimandrita Saussure, 1893 y Eublaberus Hebard, 1920 (Blattodea: Blaberidae) para México
}

\author{
First records of the genera Archimandrita Saussure, 1893 and Eublaberus Hebard, 1920 (Blattodea: \\ Blaberidae) from Mexico
}

\author{
Julio C. Estrada-Álvarez ${ }^{* 1,2}$ y César A. Guadarrama R. ${ }^{3}$ \\ ${ }^{1}$ Museo Universitario de Historia Natural "Dr. Manuel M. Villada", Universidad Autónoma del Estado de Méxi- \\ co, Instituto Literario No. 100, Colonia Centro, C.P. 50000, Toluca, Estado México. ${ }^{2}$ Entomological Research, \\ Metepec, Estado México. Email: micraten@yahoo.com.mx; ${ }^{3}$ FUCESA, Melchor Ocampo s/n, Col. Buenavista, \\ Toluca, Estado de México, México. Email: cesar@fucesa.com.mx
}

El orden Blattodea sensu lato (excepto Epifamilia Termitoidae Latreille, 1802, en Beccaloni and Eggleton 2011, 2013; Beccaloni 2014) o Suborden Blattaria Burmeister, 1829 (Blattodea menos Isoptera sensu Klass and Meier, 2006, en Li 2019), habitan en todo el planeta, excepto la Antártida, con mayor diversidad en los trópicos y subtrópicos (Djernæs 2018); se han descrito más de 4000 especies en todo el mundo (Beccaloni 2014), en el continente Americano se han reportado y/o descrito 1576 especies (Beccaloni 2014; Beccaloni and Eggleton 2011, 2013), de la cuales poco más de 800 especies se distribuyen el Neotrópico (Djernæs 2018).

Los géneros Archimandrita Saussure, 1893 y Eublaberus Hebard, 1920 según la bibliografía tienen su límite septentrional en Centroamérica, Archimandrita en Guatemala, Coatepeque (Princis, 1948) y Eublaberus en Guatemala, La Conquista [=Guatemala, Depto. San Marcos, La Conquista, localidad enmendada] (Princis, 1948).

Al revisar los ejemplares de Blattodea de la Colección Nacional de Insectos (CNIN) del Instituto de Biología (IBUNAM), de la Universidad Nacional Autónoma de México (UNAM), con el fin de conocer su diversidad, nos percatamos que tres especies de cucarachas, no están reportadas para el territorio mexicano (Estrada-Álvarez 2013).

La determinación del material se realizó conforme a Roth (1970).

Archimandrita marmorata (Stoll, 1813) (Figs. 1, 4).

Distribución conocida: Nicaragua, Costa Rica, Panamá, Colombia, Brasil, Republica Dominicana, Jamaica, Trinidad y Tobago (Beccaloni 2014; Fisk 1971; Princis 1948).

PRIMEROS REGISTROS NACIONALES: 19 Yaxchilán, Chiapas; 00/IX/1965; A. Díaz col. (CNIN). 1 ㅇ Ruinas de Bonampak, México; 03/V/1978; E. Barrera Col. (CNIN). 19 Agua Azul, Chiapas, México; 02/V/1978; E. Barrera Col. (CNIN). 10 Méx: Pue. Mesa de San Diego;
10/IX/1953; Col. Anónimo [sin cabeza] (CNIN).

Archimandrita tessellata Rehn, 1903 (Figs. 2, 4).

Distribución conocida: Guatemala, Nicaragua, Costa Rica, Panamá, Colombia (Beccaloni 2014; Fisk 1971; Maes 1992; Princis 1948; Rehn 1903).

PRIMER REGISTRO NACIONAL: $1+$ Mapastepec, Chis.; 27/VIII/1954; Col. Anónimo [Ex Colección de C. Bolívar] (CNIN).

NUEVO REGISTRO GUATEMALA: 10 Guatemala, Km. 25.5 Guatemala-San Salvador; 24/VIII/1985; E. Barrera, F. Arias, H. Velazco Cols. (CNIN).

Eublaberus distanti (Kirby, 1903) (Figs. 3, 5).

Distribución cococida: Guatemala, Nicaragua, Costa Rica, Panamá, Colombia, Brasil. Antillas (Beccaloni 2014; Evangelista et al. 2015; Fisk 1971; Hebard 1920, 1921, 1929a, 1929b, 1933; Maes 1992; Princis 1948, 1950; Rehn 1905; Rocha e Silva Albuquerque y Gurney 1962).

PRIMEROS REGISTROS NACIONALES: 10 Los Tuxtlas, Veracruz, México; 12/IV/1975; Col. Anónimo (CNIN); 10 Méx: Pue. Mesa de San Diego; 10/IV/1953; Col. Anónimo (CNIN). 1 q Pto. Eligio, Oax.; IV/1964; Col. Anónimo (CNIN). 9 Ruinas de Bonampak, Chiapas, México; 03/V/1978; E. Barrera Col. (CNIN).

\section{AGRADECIMIENTOS}

Externamos nuestra gratitud al Dr. Alejandro Zaldívar R., M. en C. Ma. Cristina Mayorga M. y M. en C. Enrique Mariño P. (CNIN-IBUNAM, UNAM, CDMX, México), por las facilidades otorgadas para la revisión de material incluido en este estudio. A los dos revisores anónimos, que contribuyeron con comentaros y correcciones. A Entomological Research y FUCESA, por financiar esta investigación.

\section{LITERATURA CITADA}

Beccaloni, G.W. and P. Eggleton. 2011. Order Blattodea Brunner von Wattenwyl, 1882 (pp. 199-200). In: Zhang, Z.-Q. (Ed.). Animal biodiversity: An outline of high- 
er-level classification and survey of taxonomic richness. Zootaxa, (3148): 1-237.

Beccaloni, G.W. and P. Eggleton. 2013. Order Blattodea (pp. 46-48). In: Zhang, Z.-Q. (Ed.) Animal Biodiversity: An outline of higher-level classification and survey of taxonomic. richness (Addenda 2013). Zootaxa, (3703): $1-82$.

Beccaloni, G.W. 2014. Cockroach Species File Online. Version 5.0/5.0. World Wide Web electronic publication. http://Cockroach.SpeciesFile.org. Fecha de consulta: 03 de diciembre de 2019.

Djernæs, M. 2018. Biodiversity of Blattodea-the Cockroaches and Termites, Chapter 14 (pp. 359-387). In: Foottit R.G. and P.H. Adler (Eds.). Insect Biodiversity: Science and Society, Vol. II. UK.

Estrada-Álvarez, J.C. 2013. Primera lista de las Cucarachas (Insecta: Dictyoptera: Blattaria) de México. Boletín de la Sociedad Entomológica Aragonesa, (53): 267-284.

Evangelista, D.A., K. Chan, K.L. Kaplan, M.M. Wilson and J.L. Ware. 2015. The Blattodea s. s. (Insecta, Dictyoptera) of the Guiana Shield. ZooKeys, (475): 37-87.

Fisk, F.W. 1971. An annotated check list of Costa Rican cockroaches (Dictyoptera: Blattaria). Proceedings of the Entomological Society of Washington, 73(4): 431444.

Hebard, M. 1920. The Blattidae of Panama. Memoirs of the American Entomological Society, (4): 1-148.

Hebard, M. 1921. Studies in the Dermaptera and Orthoptera of Colombia. Second Paper. Dermaptera and Orthopterous Families Blattidae, Mantidae and Phasmidae. Transactions of the American Entomological Society, 47(2): 107-169.

Recibido: 6 de agosto 2019

Aceptado: 2 de diciembre 2019
Hebard, M. 1929a. Previously Unreported Tropical American Blattidae (Orthoptera) in the British Museum. Transactions of the American Entomological Society, 55(4): 345-388.

Hebard, M. 1929b. Supplementary Notes on Panamanian Dermaptera and Orthoptera. Transactions of the American Entomological Society, 55(4): 389-400.

Hebard, M. 1933. Notes on Panamanian Dermaptera and Orthoptera. Transactions of the American Entomological Society, 59(2): 103-144.

Hopkins, H. 2014. A revision of the genus Arenivaga (Rehn) (Blattodea, Corydiidae), with descriptions of new species and key to the males of the genus. ZooKeys, (384): 1-256.

Li, X. 2019. Disambiguating the scientific names of cockroaches. Palaeoentomology, 2(4): 390-402.

Maes J.M. 1992. Catálogo de los Blattodea (Dictyoptera) de Nicaragua. I. Familias Polyphagidae y Blaberidae. Revista Nicaraguense de Entomología, (19): 21-28.

Morrone, J.J. y J. Márquez. 2008. Biodiversity of Mexican terrestrial arthropods (Arachnida and Hexapoda): a biogeographical puzzle, Acta Zoológica Mexicana (nueva serie), 24(1): 15-41.

Princis, K. 1948. Über einige neue bzw. wenig bekannte Blattarien aus dem Naturhistorischen Reichsmuseurn zu Stockholm. Arkiv för zoologi, 41 A(3): 1-23.

Princis, K. 1950. On a Collection of Blattaria in the Zoological Museum of the University of Oslo. Norsk entomologisk Tidsskrift, (8):129-134.

Rehn, J. A. G. 1903. Studies in American Blattiae. Transactions of the American Entomological Society, 29(3): 259-290.

Rehn, J. A. G. 1905. Notes on the Orthoptera of Costa Rica, with Descriptions of New Species. Proceedings of the Academy of Natural Sciences of Philadelphia, (57): 790-843.

Rocha e Silva-Albuquerque, I. y A.B. Gurney. 1962. Insecta Amapaensia. Report on a collection of cockroaches from the territory of Amapa, Brasil (Orthoptera, Blattoidea). Studia Entomologica, (5): 235-256.

Roth, L.M. 1970. The Male Genitalia of Blattaria. IV. Blaberidae: Blaberinae. Psyche, (77): 308-342. 

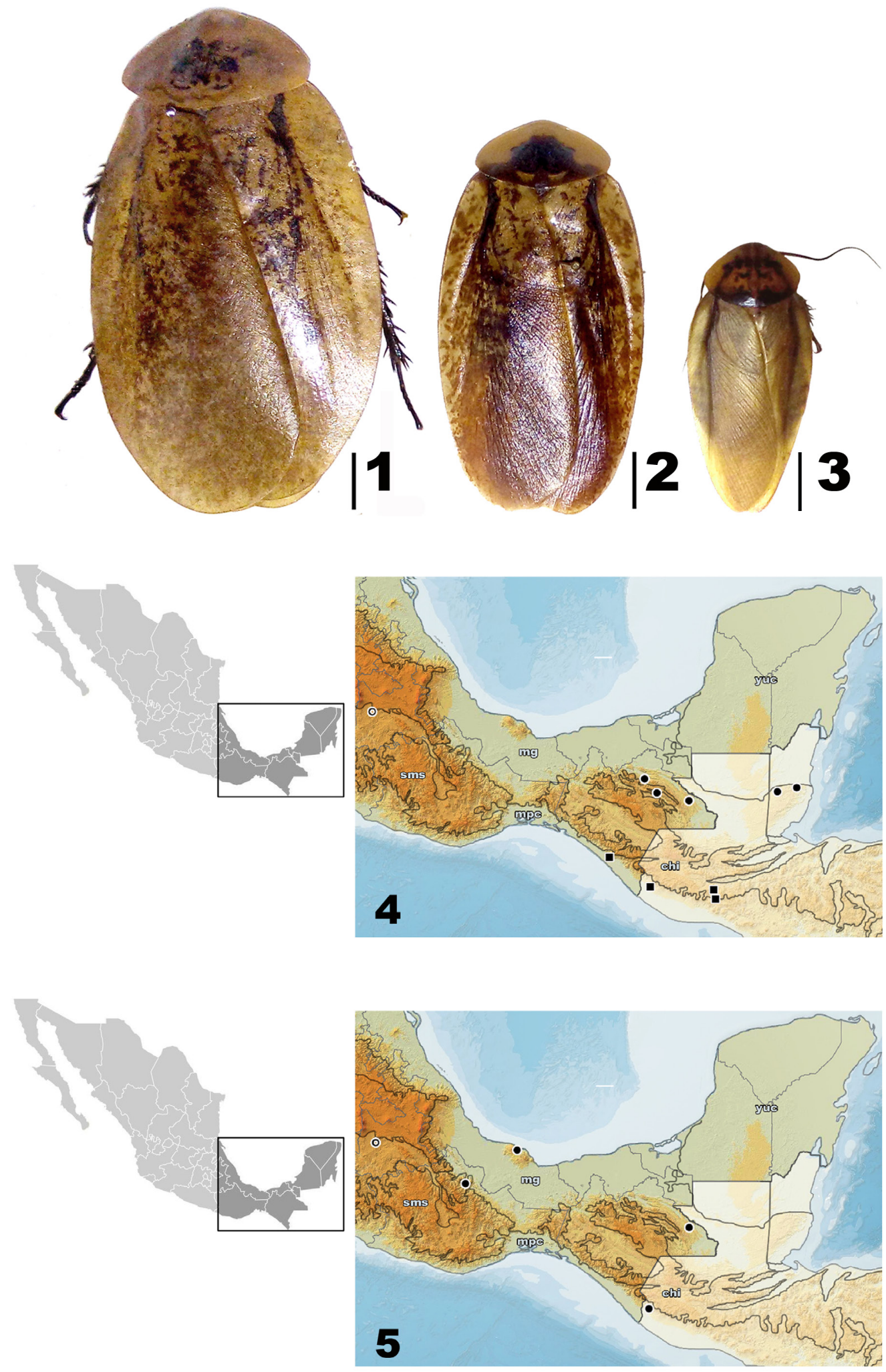

Fig. 1) Archimandrita marmorata (Stoll, 1813). Barra=10 mm. Fig. 2) Archimandrita tessellata Rehn, 1903. Barra=10 mm. Fig. 3) Eublaberus distanti (Kirby, 1903). Barra=10 mm. Fig. 4) Distribución de Archimandrita spp. en México y áreas limítrofes; PUNTOS A. marmorata (Stoll, 1813); CUADRO A. tessellata Rehn, 1903. Circulo registro dudoso de A. marmorata en Mesa de San Diego.

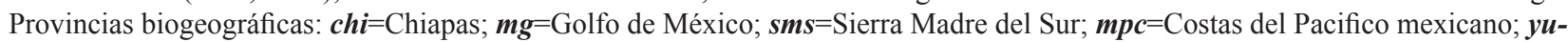
$\boldsymbol{c}=$ Yucatán (según Morrone y Márquez 2008). Fig. 5) Distribución de Eublaberus distanti (Kirby, 1903) en México y áreas limítrofes. Circulo registro dudoso para Mesa de San Diego. Provincias biogeográficas: $\boldsymbol{c h i}=$ Chiapas; $\boldsymbol{m} \boldsymbol{g}=$ Golfo de México; $\boldsymbol{s} \boldsymbol{m} \boldsymbol{s}=$ Sierra Madre del Sur; $\boldsymbol{m} \boldsymbol{p} \boldsymbol{c}=$ Costas del Pacifico mexicano; $\boldsymbol{y} \boldsymbol{u c}=$ Yucatán (según Morrone y Márquez 2008). 